\title{
Dental implants: a potential cause of bone marrow edema in the jaw-preliminary report
}

\author{
Hirotaka Muraoka* D, Naohisa Hirahara, Kotaro Ito, Takumi Kondo, Shungo Ichiki and Takashi Kaneda
}

\begin{abstract}
Background: Different magnetic resonance imaging (MRI) sequences are frequently used to examine bone marrow in the jaw, including short tau inversion recovery (STIR). MRI is a sensitive method for detecting bone marrow lesions. Currently, pantomography and computed tomography (CT) are used frequently for preoperative dental implant treatment. However, no study has evaluated bone marrow edema around dental implants using MRI. This study aimed to assess bone marrow edema in the jaw around dental implants using brain magnetic resonance images.

Methods: This retrospective cohort study was approved by our university ethics committee (EC19-011). A total of 17 patients (170 sites) who underwent brain MRI between April 2010 and March 2016 were analyzed. All subjects underwent scanning more than 3 years after implant placement. This study investigated two bone marrow signals (with implant site and without implant site). These two groups were then compared using Fisher's exact test. The Mann-Whitney $U$ test was used to analyze bone marrow signal intensity as the dependent variable and the long and short-axis diameters of the implant as the independent variables.
\end{abstract}

Results: The were 22/31 sites (71\%) and 38/139 sites (27\%) of bone marrow edema in the dental implants and without dental implants groups, respectively $(p<0.001)$. Furthermore, there was a significant correlation between bone marrow signal intensity and the short-axis diameter of the implant $(p<0.001)$.

Conclusion: The signal intensity in the bone marrow sites in the jaw with dental implants was significantly higher than that in the sites without dental implants. The present study findings suggest that dental implants are a potential cause of bone marrow edema in the jaw.

Keywords: MRI, Dental implants, Bone marrow edema, Jaw

\section{Background}

Dental implant prostheses are viable treatment options for both younger and older patients. To perform dental implant treatment, dentists need to know the precise height, width, and contour of the alveolar bone, as well as its relationship with the maxillary sinus and mandibular canal. In clinical settings and for postoperative evaluation, pantomography and computed tomography (CT)

\footnotetext{
* Correspondence: muraoka.hirotaka@nihon-u.ac.jp

Department of Radiology, Nihon University School of Dentistry at Matsudo, 2-870-1 Sakaecho-Nishi, Matsudo, Chiba 271-8587, Japan
}

are frequently used to evaluate the morphology and quality of the jawbone, and there are many reports about the usefulness of these modalities [1-3].

Bone marrow is a rich cellular connective tissue contained within the bones. Various diseases, such as anemia, inflammatory diseases, leukemia, lymphoma, and metastatic malignant tumors, greatly affect bone marrow and bone function $[4,5]$. Moreover, periimplantitis, affecting the bone marrow around dental implants, causes long-term implant failure, with consequent loss of the implant and the surrounding bone. In recent years, many reports have shown that peri-

\section{Springer Open}

(c) The Author(s). 2021 Open Access This article is licensed under a Creative Commons Attribution 4.0 International License, which permits use, sharing, adaptation, distribution and reproduction in any medium or format, as long as you give appropriate credit to the original author(s) and the source, provide a link to the Creative Commons licence, and indicate if changes were made. The images or other third party material in this article are included in the article's Creative Commons licence, unless indicated otherwise in a credit line to the material. If material is not included in the article's Creative Commons licence and your intended use is not permitted by statutory regulation or exceeds the permitted use, you will need to obtain permission directly from the copyright holder. To view a copy of this licence, visit http://creativecommons.org/licenses/by/4.0/. 
implantitis, oral squamous cell carcinoma, and bisphosphonate-related osteonecrosis of the jaw (BRONJ) are associated with dental implants [6-9]. Recently, magnetic resonance imaging (MRI) has proven to be a sensitive modality, and owing to its superior spatial resolution, it could assess the bone marrow. MRI is useful for the early detection and assessment of pathologies such as periodontitis and osteomyelitis. However, little attention has been given to the MRI-based evaluation of jawbone marrow signals around dental implants in otherwise healthy patients.

The purpose of this study was to assess bone marrow edema in the jaw around dental implants using MRI.

\section{Methods}

The was a retrospective cohort study and was approved by our university ethics committee (EC19-011). It included 17 patients (12 men, 5 women; 44-77 years of age, mean age, 63.94 years) who underwent brain MRI at the Nihon University School of Dentistry Hospital, Matsudo, Chiba, Japan, from April 2010 to March 2016. Study subjects without implants sites had no clinical findings, such as bleeding on probing (BOP), probingpocket depth (PD) less than $4 \mathrm{~mm}$, and suppuration (SUPP) around the teeth. Dental implants $(\phi 3.5 \sim 5.5 \times 10.0 \sim 18.0 \mathrm{~mm})$ and standard implant insertion techniques were used in all subjects, with the implant sites selected 3 years after implant placement. These patients had no pain associated with their implant function, no clinical implant mobility, radiographic alveolar bone loss of less than $2.0 \mathrm{~mm}$, and no history of exudate according to the criteria of Misch et al. [10]. Exclusion criteria included presence of a significant metal artifact, history of radiotherapeutic treatment, and disease (e.g., peri-implantitis, periodontitis, apical periodontitis, tumor or cyst of the jaw) affecting the jawbone marrow.

MRI was performed using a 1.5- $\mathrm{T}$ superconductive MR scanner (Intera Achieva ${ }^{\circ} 1.5$ T Nova; Philips Medical Systems, Best, the Netherlands) and a head coil. Short tau inversion recovery (STIR) images were obtained using a spin echo sequence with a repetition time, echo time, and inversion time of 2500, 60, and $180 \mathrm{~ms}$, respectively. Other imaging parameters were set as follow: section thickness, $6 \mathrm{~mm}$; matrix, $320 \times 256$; field of view, $230 \times 195.5 \mathrm{~mm}$; and one acquisition. Images, including those of the jaw, were divided into 12 regions: bilateral anterior, bilateral premolar, bilateral molar in the jaw (Fig. 1). A total of 170 sites (31 sites with implants, 139 sites without implants) were evaluated. These data were independently analyzed by two radiology specialists. The result of Cohen's kappa statistics was interpreted as follows: values between 0 and 0.2 indicate slight agreement; 0.21-0.39, minimal agreement; 0.40-

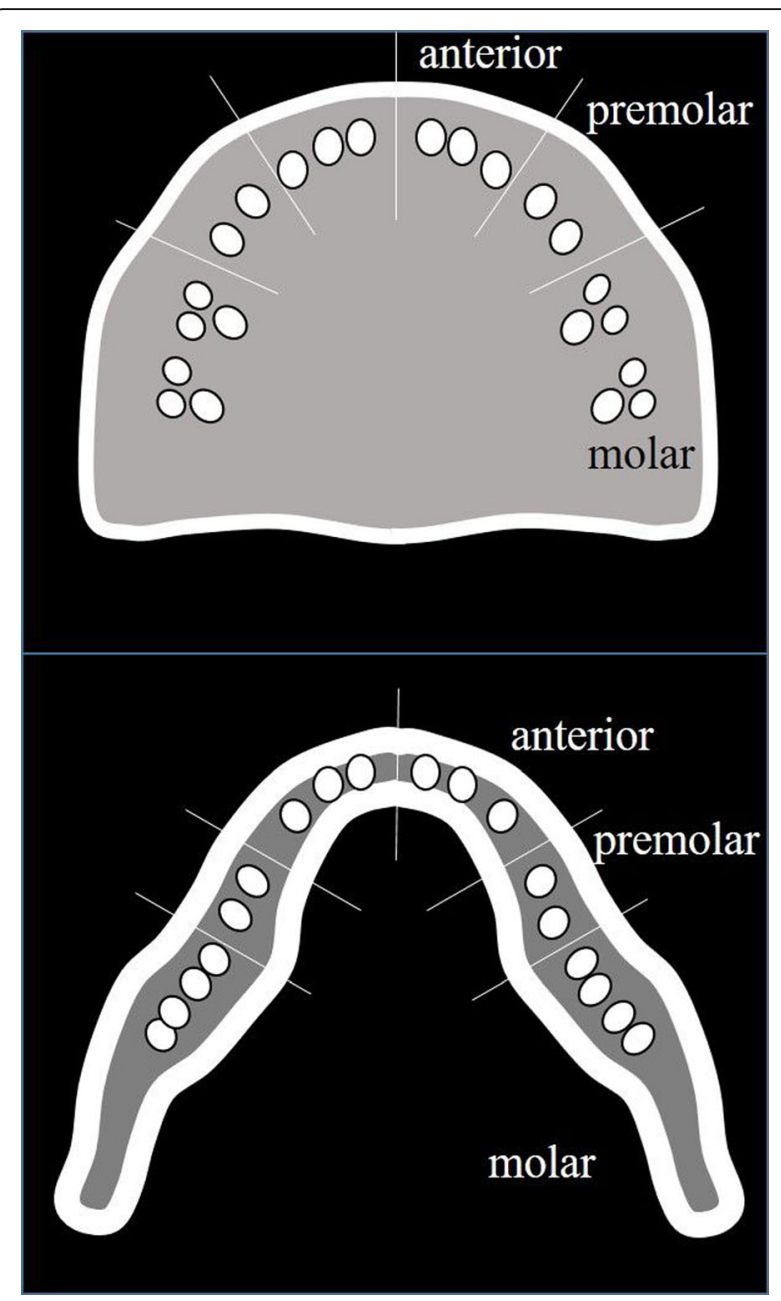

Fig. 1 Schema of 12 jaw regions in the axial MR study

0.59, weak agreement; $0.60-0.79$, moderate agreement; $0.80-0.90$, strong agreement; and $>0.90$, perfect agreement. The baseline components used to evaluate STIR MR signal intensity were cerebrospinal fluid (high signal intensity), muscle (intermediate signal intensity), and fat (low signal intensity). We classified signal intensity into five categories that included intermediate-to-high signal intensity and low-to-intermediate signal intensity in addition to high, intermediate, and low signal intensity (Fig. 2). Normal bone marrow was considered to have low signal intensity. When the bone marrow signal intensity was higher than that of fat, it was considered edematous.

The two groups (with implants site, without implants sites) were then compared using the Fisher's exact test. The Mann-Whitney $U$ test was performed using bone marrow signal intensity as the dependent variable and the long- and short-axis diameters of the implant as the independent variables. These MR signal intensity data 


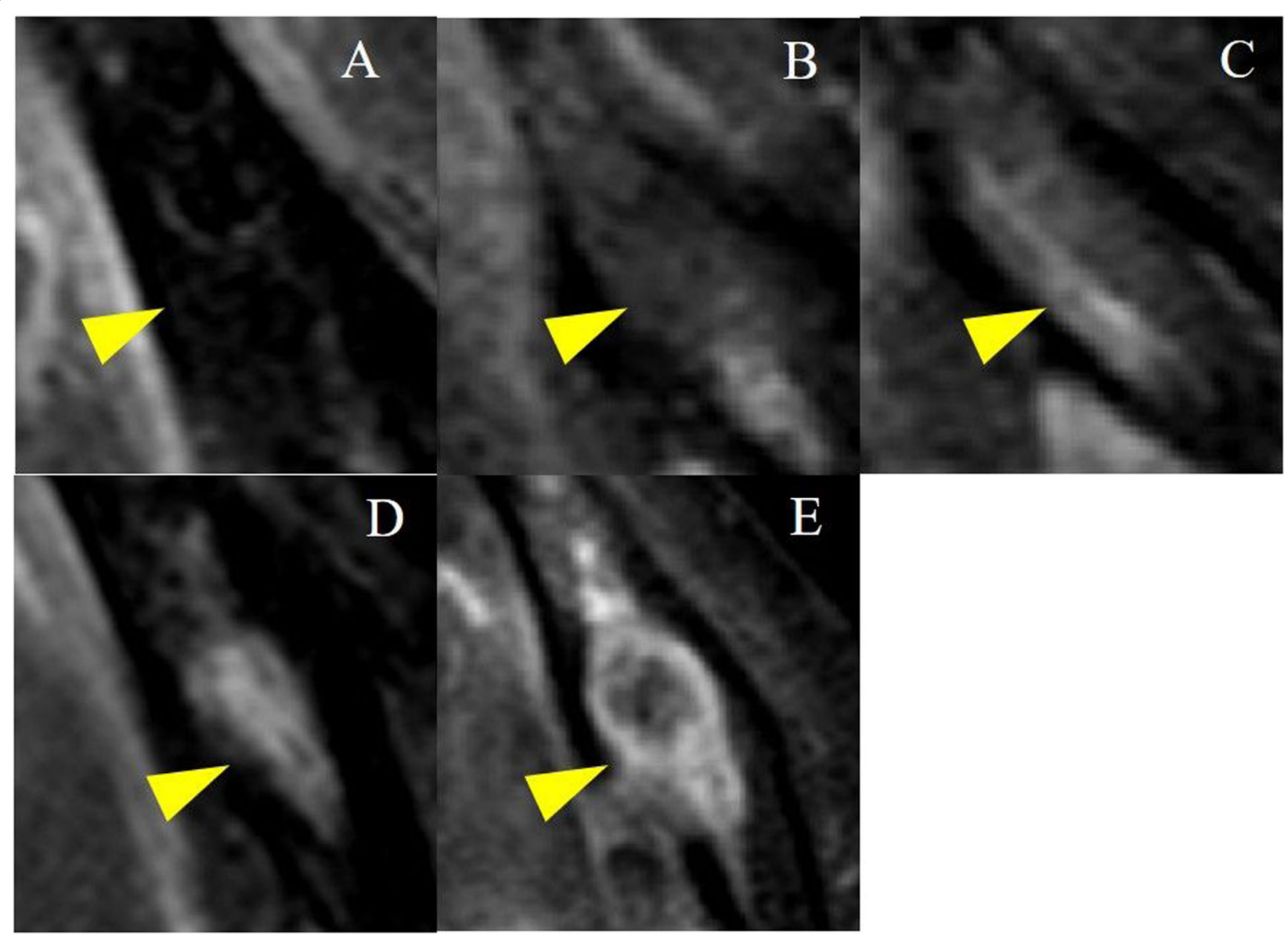

Fig. 2 The baseline structures used to determine short tau inversion recovery (STIR) sequence MR signal intensity: a fat (low-signal intensity), $\mathbf{b}$ low-to-intermediate signal intensity, c muscle (intermediate signal intensity), $\mathbf{d}$ intermediate-to-high signal intensity, and e cerebrospinal fluid (high-signal intensity) (arrowheads)

analyses were performed using a statistical package (SPSS version $21.0^{\circ}$, IBM Japan Inc., Tokyo, Japan); $p<$ 0.05 was considered to indicate significance.

\section{Results}

There was moderate agreement regarding the bone marrow status (Cohen's kappa $=0.73$ ).

Table 1 shows the details of each implant size and bone signal intensity. Table 2 shows the bone marrow status in the presence and absence of dental implants. The bone marrow signal intensity was significantly higher in the with dental implants group than in the without dental implants groups. There were no significant abnormal findings on panoramic or intraoral radiographs (Figs. 3 and 4). There were $22 / 31$ sites (71\%) and $38 / 139$ sites $(27 \%)$ with bone marrow edema in the with dental implants and without dental implants groups, respectively $(p<0.001)$.

There were $9 / 31$ sites (29\%) and 101/139 sites (73\%) without bone marrow edema in the with dental implants and without dental implants groups, respectively $(p<$ $0.01)$. All patients in the with dental implant group had no pain associated with implant function, no clinical implant mobility, less than $2.0 \mathrm{~mm}$ of radiographic crestal bone loss, and no history of exudate.

Table 3 shows the correlation between bone marrow signal intensity and the long- and short-axis diameters of the implant. There was a significant correlation between bone marrow signal intensity and the short-axis diameter of the implant $(p<0.001)$.

\section{Discussion}

In this study, significant differences were observed in the jawbone marrow signal intensity between the with dental implants and without dental implants groups.

The jaw contains a rich supply of bone marrow, which is a semi-solid tissue that may be found within the spongy or cancellous portions of bones. At birth, the mandible only contains red bone marrow; therefore, there is an overall low signal intensity on MRI. As a 
Table 1 Details of each implant size and bone signal intensity

\begin{tabular}{|c|c|c|c|}
\hline Implant No. & Bone signal intensity & Long axis diameter (mm) & Short axis diameter $(\mathrm{mm})$ \\
\hline 1 & Low & 17 & 3.5 \\
\hline 2 & Low & 14 & 3.5 \\
\hline 3 & Low & 17 & 3.5 \\
\hline 4 & Low & 14 & 4.5 \\
\hline 5 & Low & 14 & 4.1 \\
\hline 6 & Low & 13 & 4 \\
\hline 7 & Low & 15 & 4 \\
\hline 8 & Low & 16 & 4.2 \\
\hline 9 & Low & 15 & 4 \\
\hline 10 & Low-to-intermediate & 15 & 5 \\
\hline 11 & Low-to-intermediate & 18 & 4 \\
\hline 12 & Intermediate & 14 & 5 \\
\hline 13 & Intermediate & 14 & 5.5 \\
\hline 14 & Intermediate & 14 & 5.5 \\
\hline 15 & Intermediate & 14 & 5.5 \\
\hline 16 & Intermediate & 14 & 4.5 \\
\hline 17 & Intermediate & 14 & 5 \\
\hline 18 & Intermediate & 15 & 5 \\
\hline 19 & Intermediate & 15 & 5 \\
\hline 20 & Intermediate & 14 & 5 \\
\hline 21 & Intermediate & 14 & 5 \\
\hline 22 & Intermediate & 13 & 5 \\
\hline 23 & Intermediate & 13 & 5 \\
\hline 24 & Intermediate & 14 & 5.5 \\
\hline 25 & Intermediate & 17 & 4.5 \\
\hline 26 & Intermediate & 12 & 4.8 \\
\hline 27 & Intermediate & 10 & 5 \\
\hline 28 & Intermediate & 14 & 4.5 \\
\hline 29 & Intermediate & 14 & 5.5 \\
\hline 30 & Intermediate-to-high & 14 & 5 \\
\hline 31 & Intermediate-to-high & 12 & 4.7 \\
\hline 32 & Intermediate-to-high & 14 & 5.5 \\
\hline
\end{tabular}

child grows older, conversion of the red marrow to yellow marrow begins anteriorly and proceeds toward the molar regions, angle, and condyle, in that order [11]. Various diseases can greatly affect bone marrow and its function. Therefore, evaluation of bone marrow is

Table 2 Bone marrow edema in the jaw

\begin{tabular}{lllll}
\hline & \multicolumn{3}{c}{ Bone marrow edema } & \multirow{2}{*}{$\boldsymbol{P}$ values } \\
\cline { 2 - 4 } & presence & absence & Total & \\
\hline With dental implant & $22(71 \%)$ & $9(29 \%)$ & $31(18 \%)$ & $p<0.001$ \\
Without dental implant & $38(27 \%)$ & $101(73 \%)$ & $139(82 \%)$ & \\
Total & $60(35 \%)$ & $110(65 \%)$ & 170 & \\
\hline
\end{tabular}

critical for the diagnosis, treatment, and prognosis of various diseases [5]. Frequently, problems arise in determining whether an observed MRI bone marrow pattern is normal or abnormal. MRI is an important routine diagnostic tool used for imaging the oral maxillofacial area. STIR imaging represents a useful tool for the evaluation of marrow disease [12]. This study reported a characteristic high sensitivity of MRI for detecting edema. Past studies of MRI-based pre-surgical dental implant assessment have reported on the influence of dental materials on dental MRI [13-15]. However, no study has investigated the jawbone marrow around the dental implant using MRI. 


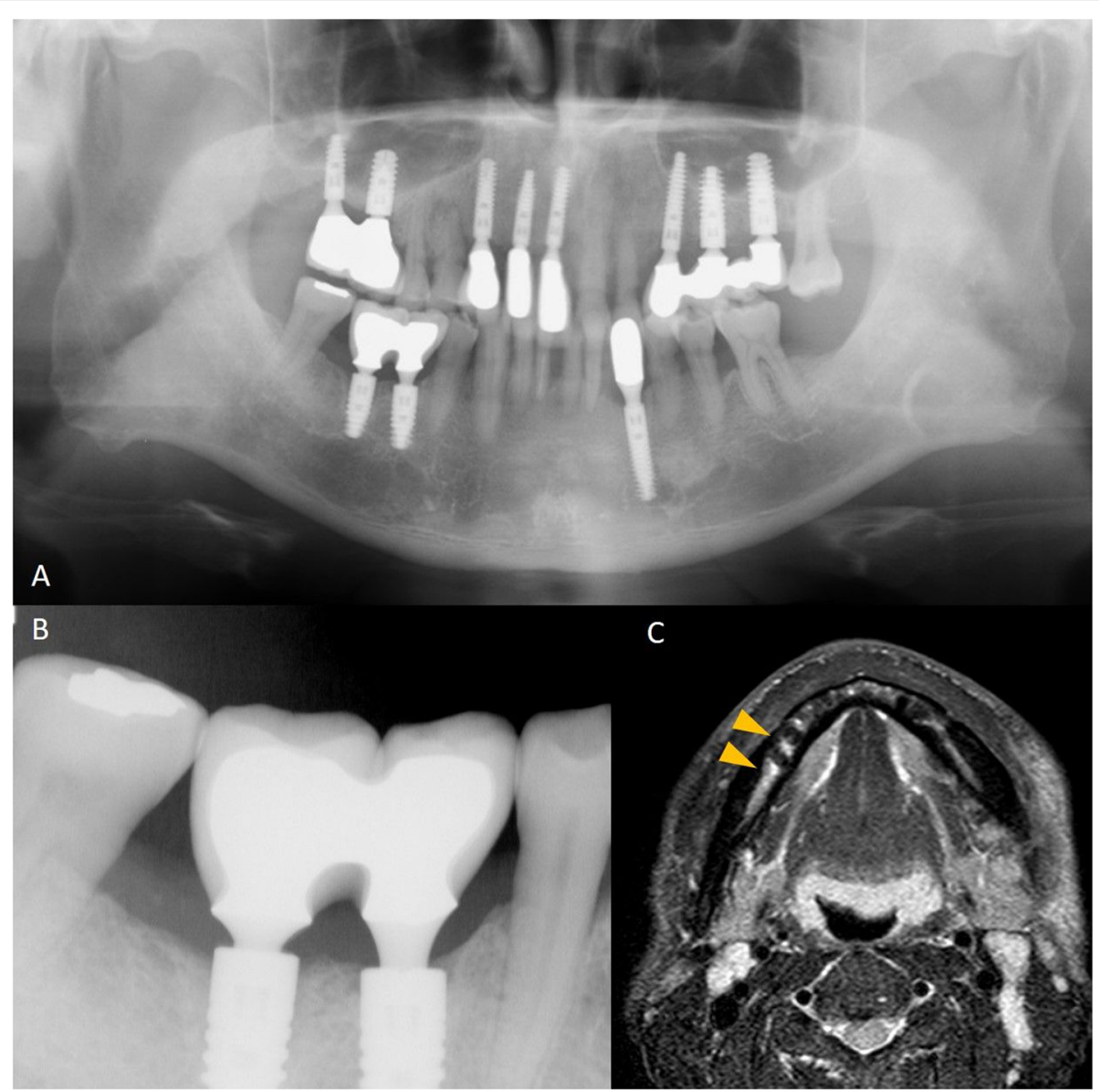

Fig. 3 A 61-year-old man with dental implants. This patient had no pain associated with implant function, no clinical implant mobility, less than $2.0 \mathrm{~mm}$ of radiographic crestal bone loss, and no history of exudate. a Panoramic radiograph shows dental implants embedded in the maxilla and mandible. $\mathbf{b}$ There are no significant abnormal findings on the intraoral radiograph (arrows). c STIR image shows a high-signal intensity on the right side of the mandibular bone marrow around dental implants (arrowheads)

Peri-implantitis is defined as an inflammatory process affecting the tissues around an osseointegrated, functional implant, resulting in the loss of the supporting bone [16]. This disease is diagnosed by changes in probing depth and radiographic evidence of bone destruction, suppuration, calculus buildup, swelling, color change, and bleeding. To diagnose an infected implant site, soft tissue measurements using probes have been suggested. Periodontitis and periimplantitis show characteristic bone marrow edema on MRI, and absence of bone marrow edema indicates that there are no abnormal clinical findings [17]. Muramatsu et al. reported bone marrow abnormalities in a high percentage of MR images of the mandibles of patients with periodontitis [18]. Generally, remarkable bone marrow edema is seen in periimplantitis with clinical findings, such as bleeding on probing and more than $2.0 \mathrm{~mm}$ of radiographic crestal bone loss (Fig. 5). In this study, characteristic bone marrow edema was seen despite the lack of clinically abnormal findings. It was considered that these changes in bone marrow edema occurred because of factors, such as the initial phase of periimplantitis and occlusal trauma despite no abnormalities observed clinically at the implant placement site. This finding also suggests that bone marrow edema may be more likely to occur in implants without a periodontal ligament with stem cells.

Second, another factor that was considered, which demonstrates spontaneous occurrence, was squamous cell carcinoma. Previous reports have indicated that squamous cell carcinoma around dental implants has been reported to cause chronic inflammation [8, 9]. Another discovery of this study was that there was a significant correlation between bone marrow signal intensity and the short-axis diameter of the implant. 


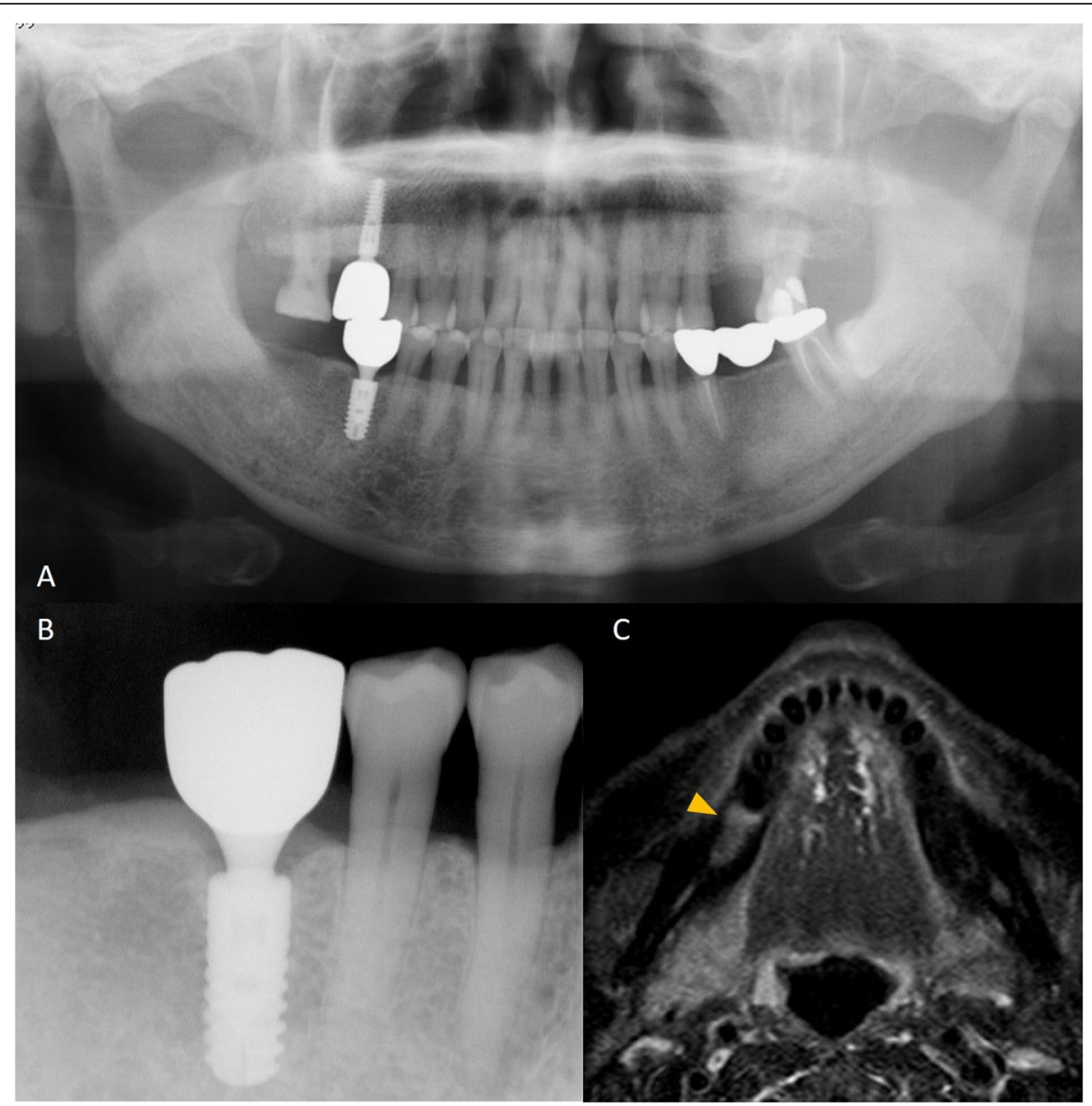

Fig. 4 A 73-year-old man with dental implants. This patient had no pain associated with implant function, no clinical implant mobility, less than $2.0 \mathrm{~mm}$ of radiographic crestal bone loss, and no history of exudate. a Panoramic radiograph shows dental implants embedded in the maxilla and mandible. $\mathbf{b}$ There are no significant abnormal findings on the intraoral radiograph (arrows). c STIR image shows a high-signal intensity on the right side of the mandibular bone marrow around the dental implant (arrowheads)

This finding may reflect that the larger the short-axis diameter, the more susceptible it is to bacterial colonization. Therefore, long-term evaluation of bone marrow is important. The present study suggested the usefulness of MRI-based examination of dental implants, and may contribute to the development of postoperative follow-up protocols.
However, our study has a few limitations. First, the sample size in our study was small due to the retrospective observational study design. We believe that long-term follow-up studies are necessary to obtain conclusive results. Moreover, STIR images are image sequences that are less affected by magnetic susceptibility artifacts; however, it was not possible to

Table 3 Relationship between the bone marrow edema and implant size in the around dental implants

\begin{tabular}{lll}
\hline & Bone marrow edema & $P$ values \\
\cline { 2 - 3 } & Presence (signal intensity = low-to-intermediate to high) & Absence (signal intensity=low) \\
\hline $\begin{array}{l}\text { Implant size } \\
\begin{array}{l}\text { Mean short axis diameter } \pm \text { SD } \\
(\mathbf{m m})\end{array}\end{array}$ & $5.0 \pm 0.39$ & $3.92 \pm 0.35$ \\
$\begin{array}{l}\text { Mean long axis diameter } \pm \text { SD } \\
(\mathbf{m m})\end{array}$ & $14.0 \pm 1.57$ & $15.0 \pm 1.41$ \\
\hline SD $=$ Standard deviation & & \\
\end{tabular}

$S D=$ Standard deviation 


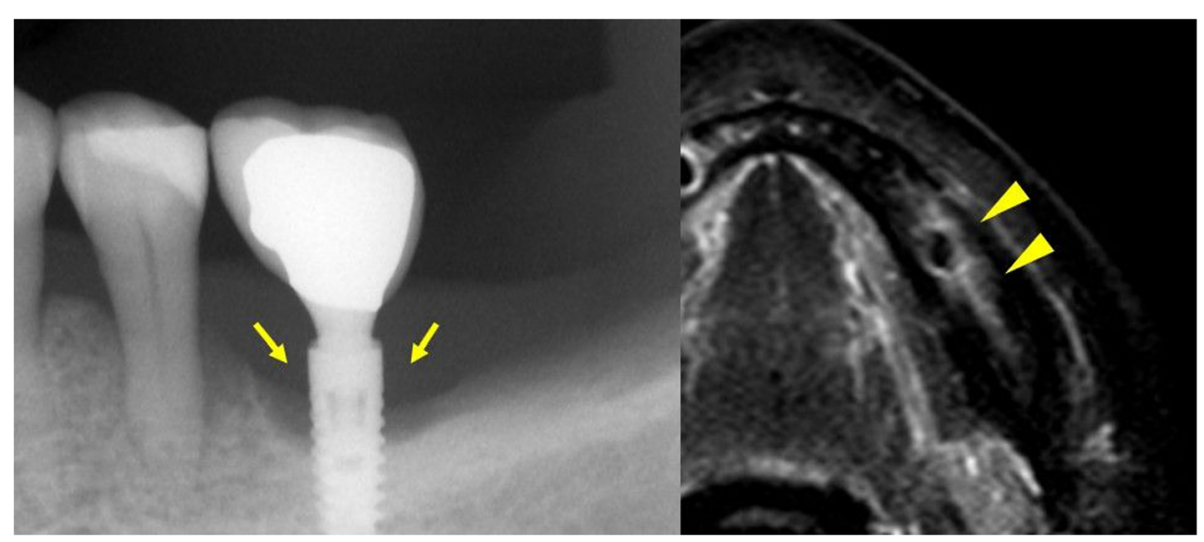

Fig. 5 A 66-year-old woman with peri-implantitis. This patient had status such as more than $2.0 \mathrm{~mm}$ of radiographically crestal bone loss (arrows), significant bone marrow edema (arrowheads)

measure the bone signal intensity in patients with severe image distortions resulting from susceptibility artifacts induced by the implant material.

In conclusion, the signal intensity in the bone marrow sites with dental implants was significantly higher than that in the sites without dental implants. The present study suggests that bone marrow edema is caused by dental implants, and that MRI may be useful for evaluating the initial phase of peri-implantitis.

\section{Abbreviations}

MRI: Magnetic resonance imaging; STIR: Short tau inversion recovery; $\mathrm{CT}$ : Computed tomography; BRONJ: Bisphosphonate-related jaw osteonecrosis; BOP: Bleeding on probing; PD: Probing-pocket depth; SUPP: Suppuration; SD: Standard deviation

\section{Acknowledgements}

There are no acknowledgments.

\section{Authors' contributions}

Hirotaka Muraoka, Kotaro Ito, and Shungo Ichiki contributed to the design and implementation of the research. Naohisa Hirahara and Takumi Kondo contributed to the analysis of the results. Hirotaka Muraoka and Takashi Kaneda contributed to the writing of the manuscript. The author(s) read and approved the final manuscript.

\section{Funding}

The authors state that this work has not received any funding.

\section{Availability of data and materials}

Not applicable.

\section{Declarations}

\section{Ethics approval and consent to participate}

The study had the approval of Nihon University ethics committee (EC19-011).

\section{Consent for publication}

Not applicable.

\section{Competing interests}

Hirotaka Muraoka, Naohisa Hirahara, Kotaro Ito, Takumi Kondo, Shungo Ichiki and Takashi Kaneda declare that they have no competing interests.
Received: 23 August 2020 Accepted: 23 February 2021

Published online: 29 March 2021

\section{References}

1. Tsukioka T, Sasaki Y, Kaneda T, Buch K, Sakai O. Assessment of relationships between implant insertion torque and cortical shape of the mandible using panoramic radiography: preliminary study. Int J Oral Maxillofac Implants. 2014;29(3):622-6.

2. Valdec S, Borm JM, Casparis S, Damerau G, Locher M, Stadlinger B. Vestibular bone thickness of the mandible in relation to the mandibular canal_a retrospective CBCT-based study. Int J Implant Dent. 2019;5:37.

3. Kassebaum DK, Nummikoski PV, Triplett RG, Langlais RP. Cross-sectional radiography for implant site assessment. Oral Surg Oral Med Oral Patho Oral Radiol Endod. 1990;70:674-8

4. Bruno CVB, Jacques M, Frederic EL, Baudouin M. Magnetic resonance imaging of the normal bone marrow. Skeletal Radiol. 1998;27:471-83.

5. Vogler JB III, Murphy WA. Bone marrow imaging. Radiology. 1988;168:679-93.

6. Chadha GK, Ahmedieh A, Kumar S, Sedghizadeh PP. Osseointegration of dental implants and osteonecrosis of the jaw in patients treated with bisphosphonate therapy: a systematic review. J Oral Implantol. 2013:39:510-20.

7. Grant BT, Amenedo C, Freeman K, Kraut RA. Outcomes of placing dental implants in patients taking oral bisphosphonates: a review of 115 cases. J Oral Maxillofac Surg. 2008;66:223-30.

8. Shaw R, Sutton D, Brown J, Cawood J. Further malignancy in field change adjacent to osseointegrated implants. Int J Oral Maxillofac Surg. 2004;33(4): 353-5.

9. Block MS, Scheufler E. Squamous cell carcinoma appearing as peri-implant bone loss: a case report. J Oral Maxillofac Surg. 2001;59(11):1349-52.

10. Misch CE, Perel ML, Wang HL, Sammartino G, Galindo-Moreno P, Trisi P, Steigmann M, Rebaudi A, Palti A, Pikos MA, Schwartz-Arad D, Choukroun J, Gutierrez-Perez JL, Marenzi G, Valavanis DK. Implant success, survival, and failure: the International Congress of Oral Implantologists (ICOI) Pisa Consensus Conference. Implant Dent. 2008;17:5-15.

11. Kaneda T, Minami M, Ozawa K, Akimoto Y, Okada H, Yamamoto H, Suzuki $H$, Sasaki Y. Magnetic resonance appearance of bone marrow in the mandible at different ages. Oral Surg Oral Med Oral Pathol Oral Radiol Endod. 1996;82: 229-33.

12. Jones K, Unger E, Granstrom P, Seeger J, Carmody R, Yoshino M. Bone marrow imaging using STIR at 0.5 Tesla and 1.5 Tesla. Magn Reson Imaging. 1992;10:169-76.

13. Gray CF, Redpath TW, Smith FW. Pre-surgical dental implant assessment by magnetic resonance imaging. J Oral Implantol. 1996;22:147-53.

14. Gray CF, Redpath TW, Smith FW. Low-field magnetic resonance imaging for implant dentistry. Dentomaxillofac Radiol. 1998;27:225-9.

15. Tymofiyeva O, Vaegler S, Rottner K, Boldt J, Hopfgartner AJ, Proff PC, Richter EJ, Jakob PM. Influence of dental materials on dental MRI. Dentomaxillofac Radiol. 2013;42(6):1-9. 
16. Heitz-Mayfield $L$, Mombelli A. The therapy of peri-implantitis: a systematic review. Int J Oral Maxillofac Implants. 2014;29:325-45.

17. Carranza FA. Clinical periodontology. 11th ed. Philadelphia, London, Tronto: W. B. Saunders Co:; 2011. p. 626-744.

18. Muramatsu T, Sekiya K, Ito K, Kawashima Y, Muraoka H, Sakae T, Okada H, Kaneda T. Mandibular bone marrow edema caused by periodontitis on magnetic resonance imaging. J Hard Tissue Biol. 2016:25:63-8.

\section{Publisher's Note}

Springer Nature remains neutral with regard to jurisdictional claims in published maps and institutional affiliations.

Submit your manuscript to a SpringerOpen ${ }^{\circ}$ journal and benefit from:

- Convenient online submission

- Rigorous peer review

- Open access: articles freely available online

High visibility within the field

- Retaining the copyright to your article

Submit your next manuscript at $\boldsymbol{\nabla}$ springeropen.com 